\title{
ASYMPTOTIC PROPERTIES OF FEEDBACK SOLUTIONS FOR A CLASS OF QUANTUM CONTROL PROBLEMS*
}

\author{
KAZUFUMI ITO ${ }^{\dagger}$ AND KARL KUNISCH ${ }^{\ddagger}$
}

\begin{abstract}
Control of quantum systems described by the Schrödinger equation is considered. Feedback control laws are developed for orbit tracking via controlled Hamiltonians, and their asymptotic properties are analyzed. Numerical integration via time splitting is also investigated and used to demonstrate the feasibility of the proposed feedback laws.
\end{abstract}

Key words. feedback control, asymptotic tracking, Schrödinger equations

AMS subject classifications. 93B11, 93B52, 49N35

DOI. $10.1137 / 080720784$

1. Introduction. Consider a quantum system with internal Hamiltonian $\mathcal{H}_{0}$ prepared in the initial state $\Psi_{0}(x)$, where $x$ denotes the relevant spatial coordinate. The state $\Psi(x, t)$ satisfies the time-dependent Schrödinger equation (we set $h=1$ ). In the presence of an external interaction taken as an electric field modeled by a coupling operator with amplitude $\epsilon(t) \in \mathbb{R}$ and a time-independent dipole moment operator $\mu$, the new Hamiltonian $\mathcal{H}=\mathcal{H}_{0}+\epsilon(t) \mu$ gives rise to the following dynamical system to be controlled:

$$
i \frac{\partial}{\partial t} \Psi(x, t)=\left(\mathcal{H}_{0}+\epsilon(t) \mu\right) \Psi(x, t), \quad \Psi(x, 0)=\Psi_{0}(x),
$$

where $\mathcal{H}_{0}$ is a positive, closed, self-adjoint operator in the Hilbert space $H$, and $\mu \in \mathcal{L}(H)$ is self-adjoint. Let $X$ be the complexified Hilbert space corresponding to $H$. Throughout we normalize the initial state by $\left|\Psi_{0}\right|_{X}=1$.

We consider the control problem of driving the state $\Psi(t)$ of (1.1) to an orbit $\mathcal{O}$ of the uncontrolled dynamics

$$
i \frac{d}{d t} \mathcal{O}(t)=\mathcal{H}_{0} \mathcal{O}(t)
$$

specifically to the one that corresponds to an eigenstate or the manifold generated by finite many eigenstates; see (1.5). An element $\psi \in \operatorname{dom}\left(\mathcal{H}_{0}\right)$ is an eigenstate of $\mathcal{H}_{0}$ if $\mathcal{H}_{0} \psi=\lambda \psi$ for $\lambda>0$. Then the corresponding orbit is given by

$$
\mathcal{O}(t)=e^{-i(\lambda t-\theta)} \psi
$$

where $\theta \in[0,2 \pi)$ is the phase factor. We have $|\mathcal{O}(t)|_{X}=1$ if $\psi$ is normalized as $|\psi|_{H}=1$. We assume that the family of eigenfunctions $\left\{\psi_{k}\right\}_{k=1}^{\infty}$ forms an orthonormal

${ }^{*}$ Received by the editors April 9, 2008; accepted for publication (in revised form) March 24, 2009; published electronically July 17, 2009.

http://www.siam.org/journals/sicon/48-4/72078.html

$\dagger$ Department of Mathematics, North Carolina State University, Raleigh, NC 27695-8205 (kito@ math.ncsu.edu). This author's research was partially supported by the Army Research Office under grant DAAD19-02-1-0394.

‡Institut für Mathematik, Karl-Franzens-Universität Graz, A-8010 Graz, Austria (karl.kunisch@ uni-graz.at). This author's research was supported in part by the Fonds zur Förderung der wissenschaftlichen Forschung under SFB 32, "Mathematical Optimization and Applications in the Biomedical Sciences." 
basis of $\mathcal{H}_{0}$ and that the associated distinct eigenvalues $\lambda_{k}$ are arranged in increasing order.

We employ a variational approach based on either of the two Lyapunov functionals

$$
\begin{array}{r}
V_{1}(\Psi(t), \mathcal{O}(t))=\frac{1}{2}|\Psi(t)-\mathcal{O}(t)|_{X}^{2}, \\
V_{2}(\Psi(t), \mathcal{O}(t))=\frac{1}{2}\left(1-\left|(\mathcal{O}(t), \Psi(t))_{X}\right|^{2}\right) .
\end{array}
$$

These variational procedures were previously discussed in [BCMR, MRT] for finite dimensional systems, for example. In connection with the functional $V_{2}$, we shall consider in section 5.3 also the case

$$
\mathcal{O}(t)=\sum_{k=1}^{N} \hat{\alpha}_{k} e^{-i\left(\lambda_{k} t-\hat{\theta}_{k}\right)} \psi_{k},
$$

where $\left\{\left(\lambda_{k}, \psi_{k}\right)\right\}_{k=1}^{N}$ are the first $N$ eigenpairs of $\mathcal{H}_{0}$ and $\sum_{k=1}^{N} \hat{\alpha}_{k}^{2}=1$.

We shall see in section 2 that $|\Psi(t)|_{X}=1$ for all $t \geq 0$. Together with $|\mathcal{O}(t)|_{X}=1$ this implies that the first functional can equivalently be expressed as

$$
V_{1}(\Psi(t), \mathcal{O}(t))=1-\operatorname{Re}(\mathcal{O}(t), \Psi(t))_{X}
$$

The second functional is motivated by the fact that

$$
V_{2}(\Psi, \mathcal{O})=0 \text { if and only if } \Psi=e^{i \theta} \mathcal{O},
$$

where the phase $\theta \in[0,2 \pi)$ is arbitrary. As a consequence we shall choose timeindependent targets and set $\mathcal{O}(t)=\mathcal{O}$ for the functional $V_{2}$. It will be shown that

$$
\frac{d}{d t} V_{1}(\Psi(t), \mathcal{O}(t))=\epsilon(t) \operatorname{Im}(\mathcal{O}(t), \mu \Psi(t))_{X} .
$$

Thus, if we set

$$
\epsilon(t)=-\frac{1}{\beta} \operatorname{Im}(\mathcal{O}(t), \mu \Psi(t))_{X}=F_{1}(\Psi(t), \mathcal{O}(t)),
$$

with weight $\beta>0$, then

$$
\frac{d}{d t} V_{1}(\Psi(t), \mathcal{O}(t))=-\beta|\epsilon(t)|^{2}
$$

Similarly, we have

$$
\frac{d}{d t} V_{2}(\Psi(t), \mathcal{O})=\epsilon(t) \operatorname{Im}\left(\overline{(\mathcal{O}, \Psi(t))_{X}}(\mathcal{O}, \mu \Psi(t))_{X}\right) .
$$

If we let

$$
\epsilon(t)=-\frac{1}{\beta} \operatorname{Im}\left(\overline{(\mathcal{O}, \Psi(t))_{X}}(\mathcal{O}, \mu \Psi(t))_{X}\right)=F_{2}(\Psi(t), \mathcal{O}),
$$

then similarly as above

$$
\frac{d}{d t} V_{2}(\Psi(t), \mathcal{O})=-\beta|\epsilon(t)|^{2}
$$

Copyright (c) by SIAM. Unauthorized reproduction of this article is prohibited. 
Note that $F_{1}$ is a linear feedback control law, whereas $F_{2}$ is quadratic. In this paper we analyze these two feedback laws with respect to their asymptotic tracking properties. Sufficient conditions will be obtained which guarantee orbit tracking for functional $V_{1}$ and manifold tracking for $V_{2}$. The latter property is the natural behavior in view of (1.7).

In order to obtain improved tracking capability we shall also analyze multiple control potentials of the form

$$
\tilde{\mu}(t)=\sum_{j=1}^{m} \epsilon_{j}(t) \mu_{j}
$$

at the end of section 5.1.

Section 2 is devoted to wellposedness of the dynamical system in open and closed loop form. In section 3 it is shown that the feedback law $F_{1}$ is optimal in the sense that $\epsilon(t)=F_{1}(\Psi(t), \mathcal{O}(t))$ minimizes

$$
\int_{0}^{T} \frac{\beta}{2}\left(|\epsilon|^{2}+\left|F_{1}(\Psi(t), \mathcal{O}(t))\right|^{2}\right) d t+V_{1}(\Psi(T), \mathcal{O}(T)) .
$$

An operator splitting method for solving (1.1) is discussed in section 4. Section 5 is devoted to analyzing the asymptotic tracking properties of the two feedback control laws. The case of operators with continuous spectrum is also considered there. Section 6 , finally, contains the description of some numerical experiments for orbit tracking.

The research of this paper is motivated by the strong activities and large literature in the physics and chemistry communities on the control of quantum mechanical systems. We refer, for instance, to [CGRR, S, ZSR], and the literature cited there. We also point out the work on stabilization of finite-dimensional Schrödinger systems [A] guaranteeing almost global convergence.

2. Wellposedness. Associated to the closed, positive, self-adjoint operator $\mathcal{H}_{0}$ densely defined in the Hilbert space $H$, we define the closed linear operator $A_{0}$ in $H \times H$ by

$$
A_{0}=\left(\begin{array}{cc}
0 & \mathcal{H}_{0} \\
-\mathcal{H}_{0} & 0
\end{array}\right)
$$

with $\operatorname{dom}\left(A_{0}\right)=\operatorname{dom}\left(\mathcal{H}_{0}\right) \times \operatorname{dom}\left(\mathcal{H}_{0}\right)$. Here $\Psi=\left(\Psi_{1}, \Psi_{2}\right) \in H \times H$ is identified with $\Psi=\Psi_{1}+i \Psi_{2} \in X$. We note that

$$
\left|\left(\Psi_{1}, \Psi_{2}\right)\right|_{H \times H}=|\Psi|_{X} \quad \text { and } \quad(\Phi, \Psi)_{H \times H}=\operatorname{Re}(\Phi, \Psi)_{X},
$$

and $X$ is isometrically isomorphic with $H \times H$ by means of

$$
(\Phi, \Psi)_{X}=\left(\Phi_{1}, \Psi_{1}\right)_{H}+\left(\Phi_{2}, \Psi_{2}\right)_{H}+i\left(\left(\Phi_{2}, \Psi_{1}\right)_{H}-\left(\Phi_{1}, \Psi_{2}\right)_{H}\right),
$$

with $\Phi=\Phi_{1}+i \Phi_{2}, \Psi=\Psi_{1}+i \Psi_{2}$. Furthermore $A_{0}$ is skew-adjoint, i.e.,

$$
\left(A_{0} \Psi, \hat{\Psi}\right)_{H \times H}=-\left(A_{0} \hat{\Psi}, \Psi\right)_{H \times H} \text { for all } \Psi, \hat{\Psi} \in \operatorname{dom}\left(A_{0}\right) .
$$

Thus by Stone's theorem $[\mathrm{P}], A_{0}$ generates a $C_{0}$-semigroup on $X$ and $\left|S(t) \Psi_{0}\right|_{X}=$ $\left|\Psi_{0}\right|_{X}$. Let $V=\operatorname{dom}\left(\mathcal{H}_{0}^{\frac{1}{2}}\right)$ and $X_{2}=V \times V$. Then $\mathcal{H}_{0} \in \mathcal{L}\left(V, V^{*}\right)$ with $V^{*}=$ $\operatorname{dom}\left(\mathcal{H}_{0}^{-\frac{1}{2}}\right)$ and $V$ is equipped with

$$
|\phi|_{V}^{2}=\left\langle\mathcal{H}_{0} \phi, \phi\right\rangle_{V^{*}, V}
$$

as norm. The restriction of $S(t)$ to $X_{2}$ defines a $C_{0}$-semigroup. 
Associated to the self-adjoint operator $\mu \in \mathcal{L}(H)$, we define the skew-adjoint operator

$$
B=\left(\begin{array}{cc}
0 & \mu \\
-\mu & 0
\end{array}\right) .
$$

Then for $\epsilon \in L^{2}(0, T)$ there exists a unique mild solution $\Psi(t) \in C(0, T ; X)$ to

$$
\Psi(t)=S(t) \Psi_{0}+\int_{0}^{t} S(t-s) \epsilon(s) B \Psi(s) d s, \quad t \in[0, T]
$$

and

$$
\frac{d}{d t} \Psi=A_{0} \Psi(t)+\epsilon(t) B \Psi(t) \quad \text { in }\left(\operatorname{dom}\left(A_{0}\right)\right)^{*} ;
$$

see, e.g., [BMS], [P, Chapter 4], [IK, Chapter 2]. Here $\left(\operatorname{dom}\left(A_{0}\right)\right)^{*}=\left(\operatorname{dom}\left(\mathcal{H}_{0}\right)\right)^{*} \times$ $\left(\operatorname{dom}\left(\mathcal{H}_{0}\right)\right)^{*}$, and $\left(\operatorname{dom}\left(\mathcal{H}_{0}\right)\right)^{*}$ is the topological dual of $\operatorname{dom}\left(\mathcal{H}_{0}\right)$ with respect to the pivot space $H$. Equivalently,

$$
\frac{d}{d t} \Psi(t)=-i\left(\mathcal{H}_{0} \Psi(t)+\epsilon(t) \mu \Psi(t)\right)
$$

in the complexified dual space of $\operatorname{dom}\left(\mathcal{H}_{0}\right)$. Since $\mathcal{O}(t) \in C\left(0, T ; \operatorname{dom}\left(A_{0}\right)\right) \cap C^{1}(0, T ; X)$, we have

$$
\frac{d}{d t} \mathcal{O}(t)=-i \mathcal{H}_{0} \mathcal{O}(t) \text { in } X
$$

Thus

$$
\begin{aligned}
& \frac{d}{d t} \operatorname{Re}(\mathcal{O}(t), \Psi(t))_{X}=\operatorname{Re}\left(\left(-i \mathcal{H}_{0} \mathcal{O}(t), \Psi(t)\right)_{X}+\left(\mathcal{O}(t),-i\left(\mathcal{H}_{0} \Psi(t)+\epsilon(t) \mu \Psi(t)\right)\right)_{X}\right) \\
& =\operatorname{Re}(i \epsilon(t)(\mathcal{O}(t), \mu \Psi(t)))_{X}=-\epsilon(t) \operatorname{Im}(\mathcal{O}(t), \mu \Psi(t))_{X}
\end{aligned}
$$

which proves (1.8). Similarly

$$
\begin{aligned}
& \frac{d}{d t} \frac{1}{2}\left|(\mathcal{O}(t), \Psi(t))_{X}\right|^{2}=\frac{d}{d t} \frac{1}{2}\left((\mathcal{O}(t), \Psi(t))_{X} \overline{(\mathcal{O}, \Psi)_{X}}\right) \\
= & \frac{1}{2}\left((\mathcal{O}, \Psi)_{X} \frac{d}{d t} \overline{(\mathcal{O}, \Psi)_{X}}+\frac{d}{d t}(\mathcal{O}, \Psi)_{X} \overline{(\mathcal{O}, \Psi)_{X}}\right)=\operatorname{Re}\left(\overline{(\mathcal{O}, \Psi)_{X}} \frac{d}{d t}(\mathcal{O}, \Psi)_{X}\right) \\
= & \operatorname{Re}\left(\overline{(\mathcal{O}(t), \Psi(t))_{X}}\left(\left(-i \mathcal{H}_{0} \mathcal{O}(t), \Psi(t)\right)_{X}+\left(\mathcal{O}(t),-i\left(\mathcal{H}_{0} \Psi(t)+\epsilon(t) \mu \Psi(t)\right)\right)_{X}\right)\right) \\
= & -\epsilon(t) \operatorname{Im}\left(\overline{(\mathcal{O}(t), \Psi(t))_{X}}(\mathcal{O}(t), \mu \Psi(t))_{X}\right)
\end{aligned}
$$

which proves (1.11).

Thus, we obtain the closed loop system of the form

$$
\Psi(t)=S(t) \Psi_{0}+\int_{0}^{t} S(t-s) F(\Psi(s), \mathcal{O}(s)) B \Psi(s) d s
$$

where $F$ denotes either $F_{1}$ or $F_{2}$.

Copyright $@$ by SIAM. Unauthorized reproduction of this article is prohibited. 
We show that (2.4) has a unique solution. For this purpose we consider (2.1) with $\epsilon \in C(0, T ; X)$. Let

$$
B_{\lambda}=\left(\begin{array}{cc}
R_{\lambda} & 0 \\
0 & R_{\lambda}
\end{array}\right) B\left(\begin{array}{cc}
R_{\lambda} & 0 \\
0 & R_{\lambda}
\end{array}\right)
$$

where $R_{\lambda}$ is the Yosida approximation of $-\mathcal{H}_{0}$, i.e., $R_{\lambda}=-\mathcal{H}_{0}\left(\lambda I+\mathcal{H}_{0}\right)^{-1}$. We have $\left\|R_{\lambda}\right\|_{X} \leq \frac{1}{\lambda}$ and $\lim _{\lambda \rightarrow \infty} R_{\lambda} \Psi=\Psi$, for every $\Psi \in X$. Moreover, $B_{\lambda}$ is skew-adjoint. Let $\Psi_{\lambda} \in C(0, T ; X)$ be the mild solution of

$$
\Psi_{\lambda}(t)=S(t) \Psi_{0}^{\lambda}+\int_{0}^{t} S(t-s) \epsilon(s) B_{\lambda} \Psi_{\lambda}(s) d s
$$

where $\Psi_{0}^{\lambda} \in \operatorname{dom}\left(A_{0}\right)$ with $\Psi_{0}^{\lambda} \rightarrow \Psi_{0}$ in $\mathrm{X}$ and $\left|\Psi_{0}^{\lambda}\right|_{X}=\left|\Psi_{0}\right|_{X}=1$. Then $f_{\lambda}=$ $\varepsilon B_{\lambda} \Psi \in C\left(0, T ; \operatorname{dom}\left(A_{0}\right)\right)$ and hence $\Psi_{\lambda} \in C^{1}(0, T ; X)$ is the strong solution of

$$
\left\{\begin{array}{l}
\frac{d}{d t} \Psi_{\lambda}(t)=A_{0} \Psi_{\lambda}(t)+\varepsilon(t) B_{\lambda} \Psi_{\lambda}(t), \\
\Psi_{\lambda}(0)=\Psi_{0}^{\lambda} .
\end{array}\right.
$$

This implies that

$$
\frac{1}{2} \frac{d}{d t}\left|\Psi_{\lambda}(t)\right|_{H \times H}^{2}=\left(A_{0} \Psi_{\lambda}(t)+\varepsilon(t) B_{\lambda} \Psi_{\lambda}(t), \Psi_{\lambda}(t)\right)_{H \times H}=0
$$

and hence $\left\|\Psi_{\lambda}(0)\right\|_{X}=1$ for all $t \in[0, T]$. Further

$$
\begin{aligned}
\left|\Psi_{\lambda}(t)-\Psi(t)\right|_{X} \leq & \left|S(t)\left(\Psi_{0}^{\lambda}-\Psi_{0}\right)\right|_{X}+\int_{0}^{t}\left|S(t-s) \varepsilon(s) B_{\lambda}\left(\Psi_{\lambda}(s)-\Psi(s)\right)\right|_{X} d s \\
& +\int_{0}^{t}\left|S(t-s)\left(B_{\lambda}-B\right) \Psi(s)_{X}\right| d s .
\end{aligned}
$$

Consequently there exists a constant $K$ independent of $\lambda$ such that

$$
\begin{gathered}
\left\|\Psi_{\lambda}(t)-\Psi(t)\right\|_{X} \leq K \int_{0}^{t}\left\|\Psi_{\lambda}(s)-\Psi(s)\right\|_{X} d s+\left\|\Psi_{0}^{\lambda}-\Psi_{0}\right\|_{X} \\
+\int_{0}^{t}\left\|\left(B_{\lambda}-B\right) \Psi(s)\right\| d s .
\end{gathered}
$$

By Gronwall's lemma and Lebesgue's bounded convergence theorem we have that $\Psi_{\lambda} \rightarrow \Psi$ in $C(0, T ; X)$. Consequently $\|\Psi(t)\|_{X}=1$ for all $t \in[0, T]$.

To argue that $(2.4)$ has a unique solution let $\mathcal{O} \in C(0, T ; X)$, with $\|\mathcal{O}(t)\|_{X}=1$, and consider the iteration

$$
\Psi_{n}(t)=S(t) \Psi_{0}+\int_{0}^{t} S(t-s) F\left(\Psi_{n-1}(s), \mathcal{O}(s)\right) B \Psi_{n}(s) d s
$$

which is initialized by the constant function with value $\Psi_{0}$. We use the fact that there exists a constant $M>0$ such that

$$
|F(\Psi, \mathcal{O})-F(\hat{\Psi}, \hat{\mathcal{O}})| \leq M\left(|\Psi-\hat{\Psi}|_{X}+|\mathcal{O}-\hat{\mathcal{O}}|_{X}\right)
$$

for every $(\Psi, \hat{\Psi}) \in X \times X,(\mathcal{O}, \hat{\mathcal{O}}) \in X \times X$ with $|\Psi|_{X}=|\hat{\Psi}|_{X}=|\mathcal{O}|_{X}=|\hat{\mathcal{O}}|_{X}=1$. Consequently for $\Psi_{n-1} \in C(0, T ; X)$ we have that $\epsilon=F\left(\Psi_{n-1}, \mathcal{O}\right) \in C(0, T ; X)$ 
and by the above discussion (2.5) admits a unique solution $\Psi_{n} \in C(0, T ; X)$ for each $n=1,2, \ldots$ with $\left|\Psi_{n}(t)\right|_{X}=1$ for $t \in[0, T]$. Consequently there exists a constant $K=K(|\mu|, M)$, but independent of $n$ and $t$, such that for consecutive iterates we have

$$
\begin{aligned}
& \left|\Psi_{n+1}(t)-\Psi_{n}(t)\right|_{X}=\left|\int_{0}^{t} S(t-s)\left(F\left(\Psi_{n}, \mathcal{O}\right) B \Psi_{n+1}-F\left(\Psi_{n-1}, \mathcal{O}\right) B \Psi_{n}\right)\right| d s \\
& \quad \leq \int_{0}^{t}\left(\left|F\left(\Psi_{n}, \mathcal{O}\right) B\left(\Psi_{n+1}-\Psi_{n}\right)\right|+\left|\left(F\left(\Psi_{n}, \mathcal{O}\right)-F\left(\Psi_{n-1}, \mathcal{O}\right)\right) B \Psi_{n}\right|\right) d s \\
& \quad \leq K \int_{0}^{t}\left|\Psi_{n+1}-\Psi_{n}\right| d s+K \int_{0}^{t}\left|\Psi_{n}-\Psi_{n-1}\right| d s
\end{aligned}
$$

By Gronwall's lemma this leads to

$$
\left|\Psi_{n+1}-\Psi_{n}\right|_{C(0, \bar{t} ; X)} \leq K \bar{t} e^{K \bar{t}}\left|\Psi_{n}-\Psi_{n-1}\right|_{C(0, \bar{t} ; X)}
$$

for each $\bar{t} \in(0, T]$. Choosing $\bar{t}$ such that $\theta=K \bar{t} e^{K \bar{t}}<1$ we obtain

$$
\left|\Psi_{n+1}-\Psi_{n}\right|_{C(0, \tau, X)} \leq \theta^{n}\left|\Psi_{1}-\Psi_{0}\right|_{C(0, T ; X)} \rightarrow 0
$$

as $n \rightarrow \infty$. Thus $\Psi_{n}$ is a Cauchy sequence in $C(0, \bar{t} ; X)$ and it follows that (2.4) has a unique solution on $[0, \tau]$. Since $K$ is independent of $n$, so is $\tau$, and hence by the continuation method (2.4) has a unique solution $\Psi \in C(0, T ; X)$.

An alternative proof for the wellposedness of the closed loop system can be based on Lipschitz perturbation theory of linear evolution equations $[\mathrm{P}]$. For the second feedback law this follows from the results in $[\mathrm{M}]$.

The existence and uniqueness results of this section also apply if the single-pole control potential $\epsilon \mu$ is replaced by a multipole control potential as in (1.14).

3. Optimality. In the introduction we argued that the feedback laws are chosen such that the Lyapunov functionals $V_{1}, V_{2}$ decay along the controlled trajectories. Now we argue that the feedback law corresponding to the first functional is also optimal in a sense to be specified below. This is a special case of a well-known procedure in feedback control which asserts that for a given Lyapunov function $\mathrm{V}$ or a feedback control law a cost functional can be constructed such that $V$ is a solution to the associated Hamilton-Jacobi equation; see, e.g., [FK] and [G]. Section 5 will be devoted to analyzing the asymptotic properties of both feedback laws.

We argue that

$$
V_{1}(\Psi, \mathcal{O}(t))=1-(\mathcal{O}(t), \Psi)_{H \times H}
$$

satisfies the Hamilton Jacobi equation

$\frac{\partial V_{1}}{\partial t}+\min _{\epsilon}\left[\frac{\beta}{2}|\epsilon|^{2}+\left(V_{1}\right)_{\Psi}\left(A_{0} \Psi+\epsilon B \Psi\right)\right]+\frac{1}{2 \beta}\left|(\mathcal{O}(t), B \Psi)_{H \times H}\right|^{2}=0 \quad$ for $\Psi \in \operatorname{dom}\left(A_{0}\right)$,

where

$$
\left(V_{1}\right)_{\Psi}(\Phi)=-(\mathcal{O}(t), \Phi)_{H \times H}
$$

In fact,

$$
\epsilon^{*}=\frac{1}{\beta}(\mathcal{O}(t), B \Psi)_{H \times H}=F_{1}(\Psi, \mathcal{O}(t))
$$

Copyright (C) by SIAM. Unauthorized reproduction of this article is prohibited. 
minimizes

$$
\frac{\beta}{2}|\epsilon|^{2}-\epsilon(\mathcal{O}(t), B \Psi)_{H \times H}
$$

This implies

$$
\begin{aligned}
\frac{\partial V_{1}}{\partial t} & +\frac{\beta}{2}\left|\epsilon^{*}(t)\right|^{2}+\left(V_{1}\right)_{\Psi}\left(A_{0} \Psi+\epsilon^{*} B \Psi\right)+\frac{1}{2 \beta}\left|(\mathcal{O}(t), B \Psi)_{H \times H}\right|^{2} \\
& =-\left(A_{0} \mathcal{O}(t), \Psi\right)_{H \times H}-\left(\mathcal{O}(t), A_{0} \Psi+\epsilon^{*}(t) B \Psi\right)_{H \times H}+\frac{1}{\beta}\left|(\mathcal{O}(t), B \Psi)_{H \times H}\right|^{2} \\
& =-\epsilon^{*}(t)(\mathcal{O}(t), B \Psi)_{H \times H}+\frac{1}{\beta}\left|(\mathcal{O}(t), B \Psi)_{H \times H}\right|^{2}=0
\end{aligned}
$$

as desired.

We next show that $\epsilon^{*}$ minimizes

$$
J(\epsilon)=\int_{0}^{T}\left(\frac{\beta}{2}|\epsilon(t)|^{2}+\frac{1}{2 \beta}\left|(\mathcal{O}(t), B \Psi(t))_{H \times H}\right|^{2}\right) d t+V_{1}(\Psi(T), \mathcal{O}(T)),
$$

over $\epsilon \in L^{2}(0, T)$.

For this purpose choose any $\epsilon \in L^{2}(0, T)$ and let $\Psi(t) \in C(0, T ; X)$ be the solution to $(2.1)-(2.2)$. Since $\mathcal{O}(t) \in C^{1}(0, T ; X) \cap C\left(0, T ; \operatorname{dom}\left(A_{0}\right)\right)$ we have

$$
\frac{d}{d t} V_{1}(\Psi(t), \mathcal{O}(t))=-\left(A_{0} \mathcal{O}(t), \Psi(t)\right)_{H \times H}-\left(\mathcal{O}(t), A_{0} \Psi(t)+\epsilon(t) B \Psi(t)\right)_{H \times H} .
$$

Integrating over $(0, T)$ and using $a b=\frac{1}{2} a^{2}+\frac{1}{2} b^{2}-\frac{1}{2}|a-b|^{2}$ we find

$$
\begin{gathered}
V_{1}(\Psi(T), \mathcal{O}(T))+\int_{0}^{T}\left(\frac{\beta}{2}|\epsilon(t)|^{2}+\frac{1}{2 \beta}\left|(\mathcal{O}(t), B \Psi(t))_{H \times H}\right|^{2}\right) d t \\
=V_{1}(\Psi(0), \mathcal{O}(0))+\int_{0}^{T} \frac{\beta}{2}\left|\epsilon(t)-\frac{1}{\beta}(\mathcal{O}(t), B \Psi(t))_{H \times H}\right|^{2} d t .
\end{gathered}
$$

Hence

$$
\epsilon^{*}(t)=\frac{1}{\beta}\left(\mathcal{O}(t), B \Psi^{*}(t)\right)_{H \times H}=F_{1}\left(\Psi^{*}(t), \mathcal{O}(t)\right)
$$

where $\Psi^{*}(t)$ is the trajectory corresponding to $\epsilon^{*}(t)$. Thus $\epsilon^{*}$ given in (3.1) minimizes $J$ over $L^{2}(0, T)$.

4. Operator splitting and numerical methods. Since the Hamiltonian is the sum of $\mathcal{H}_{0}$ and $\epsilon(t) \mu$, it is very natural to consider time integration based on the operator splitting method. For the stepsize $h>0$ consider the Lie-Trotter splitting method,

$$
\frac{\Psi^{k+1}-\hat{\Psi}^{k}}{h}=\epsilon^{k} B \frac{\Psi^{k+1}+\hat{\Psi}^{k}}{2}, \quad \hat{\Psi}^{k}=S(h) \Psi^{k},
$$

and the Strang splitting method,

$$
\begin{aligned}
& \frac{\hat{\Psi}^{k+1}-\hat{\Psi}^{k}}{h}=\epsilon^{k} B \frac{\hat{\Psi}^{k+1}+\hat{\Psi}^{k}}{2}, \quad \hat{\Psi}^{k}=S\left(\frac{h}{2}\right) \Psi^{k}, \\
& \Psi^{k+1}=S\left(\frac{h}{2}\right) \hat{\Psi}^{k+1},
\end{aligned}
$$

Copyright (C) by SIAM. Unauthorized reproduction of this article is prohibited. 
where

$$
\epsilon^{k}=\frac{1}{h} \int_{k h}^{(k+1) h} \epsilon(s) d s .
$$

For time integration of the controlled Hamiltonian we employ the Crank-Nicolson scheme since it is a norm-preserving scheme. In fact, since $B$ is skew-adjoint

$$
\left(\frac{\Psi^{k+1}-\hat{\Psi}^{k}}{h}, \Psi^{k+1}+\hat{\Psi}^{k}\right)_{X}=0,
$$

and thus $\left|\Psi^{k+1}\right|_{X}^{2}=\left|\hat{\Psi}^{k}\right|_{X}^{2}$. The Lie-Trotter splitting is of first order, whereas the Strang splitting is of second order as time integration. We refer to $[\mathrm{B}, \mathrm{IK}]$ and the literature cited there for further discussion.

Convergence of (4.1) and (4.2) is addressed in the following theorem.

Theorem 4.1. If we define $\Psi_{h}(t)=\Psi^{k}$ on $[k h,(k+1) h)$, then

$$
\left|\Psi_{h}(t)-\Psi(t)\right|_{X} \rightarrow 0 \text { uniformly in } t \in[0, T]
$$

where $\Psi(t), t \geq 0$, satisfies

$$
\Psi(t)=S(t) \Psi_{0}+\int_{0}^{t} S(t-s) \epsilon(s) B \Psi(s) d s .
$$

Proof. Define the one-step transition operator

$$
\Psi^{k+1}=T_{h}(t) \Psi^{k}
$$

by

$$
T_{h}(t) \Psi=\left(I+\frac{\epsilon^{k} h}{2} B\right)\left(I-\frac{\epsilon^{k} h}{2} B\right)^{-1} S(h) \Psi \text { for } t \in[k h,(k+1) h) .
$$

Then $\left|T_{h}(t) \Psi\right|_{X}=|\Psi|_{X}$ and

$$
A_{h}(t)=\frac{T_{h}(t) \Psi-\Psi}{h}=\frac{J_{h / 2}\left(\epsilon^{k} B\right)-I}{h / 2} S(h) \Psi+\frac{S(h) \Psi-\Psi}{h},
$$

where

$$
J_{h / 2}\left(\epsilon^{k} B\right)=\left(I-\frac{\epsilon^{k} h}{2} B\right)^{-1}
$$

Since for $\Psi \in X$

$$
\lim _{h \rightarrow 0^{+}} \frac{J_{h / 2}\left(\epsilon^{k} B\right)-I}{h / 2} \Psi=\epsilon(t) B \Psi
$$

and for $\Psi \in \operatorname{dom}(A)$

$$
\lim _{h \rightarrow 0^{+}} \frac{S(h) \Psi-\Psi}{h}=A_{0} \Psi,
$$

Copyright $\odot$ by SIAM. Unauthorized reproduction of this article is prohibited. 
it follows that for $\Psi \in \operatorname{dom}(A)$ and $\epsilon \in C(0, T)$

$$
\left.\mid A_{h}(t) \Psi-\left(A_{0} \Psi+\epsilon(t) B\right) \Psi\right)\left.\right|_{X} \rightarrow 0 \text { as } h \rightarrow 0^{+} .
$$

It thus follows from the Chernoff theorem $[\mathrm{IK}]$ that $\left|\Psi_{h}(t)-\Psi(t)\right|_{X} \rightarrow 0$ uniformly in $t \in[0, T]$. Note that

$$
\Psi^{k+1}=S(h) \Psi^{k}+h \epsilon^{k} J_{h / 2}\left(\epsilon^{k} B\right) S(h) \Psi^{k}
$$

and thus

$$
\Psi^{m}=S(m h) \Psi_{0}+\sum_{k=1}^{m} h S((m-k) h) \epsilon^{k} B J_{h / 2}\left(\epsilon^{k} B\right) S(h) \Psi^{k-1} .
$$

Thus, letting $h \rightarrow 0$ in this expression, $\Psi(t) \in C(0, T ; X)$ satisfies $(2.1)$.

For the Strang splitting

$$
T_{h}(t)=S\left(\frac{h}{2}\right)\left(I+\frac{\epsilon^{k} h}{2} B\right)\left(I+\frac{\epsilon^{k} h}{2} B\right)^{-1} S\left(\frac{h}{2}\right) \Psi .
$$

Then

$$
A_{h}(t) \Psi=\frac{T_{h}(t) \Psi-\Psi}{h}=S\left(\frac{h}{2}\right) \frac{J_{h / 2}\left(\epsilon_{k} B\right)-I}{h / 2} S\left(\frac{h}{2}\right) \Psi+\frac{S(h) \Psi-\Psi}{h} .
$$

Thus, using the same arguments we have $A_{h}(t) \Psi \rightarrow A(t) \Psi$ for $\Psi \in \operatorname{dom}(A)$. Hence it follows from the Chernoff theorem that the Strang splitting method converges.

Let $F$ denote one of our feedback laws $F_{1}$, or $F_{2}$. Suppose we select $\epsilon^{k}$ on $[k h,(k+$ 1) $h$ ) for the discrete-time systems (4.1) such that

$$
\epsilon^{k}=F\left(\Psi^{k+1 / 2}, \mathcal{O}^{k+1}\right), \quad \Psi^{k+1 / 2}=\frac{\Psi^{k+1}+\hat{\Psi}^{k}}{2} .
$$

Then $\Psi^{k}$ satisfies the closed loop system given by

$$
\begin{aligned}
& \frac{\Psi^{k+1}-\hat{\Psi}^{k}}{h}=\epsilon^{k} B \frac{\Psi^{k+1}+\hat{\Psi}^{k}}{2}, \quad \hat{\Psi}^{k}=S(h) \Psi^{k}, \\
& \epsilon^{k}=F\left(\Psi^{k+1 / 2}, \mathcal{O}^{k+1}\right) .
\end{aligned}
$$

Since

$$
V(S(h) \Psi, S(h) \mathcal{O})=V(\Psi, \mathcal{O})
$$

we have

$$
V\left(\Psi^{k+1}, \mathcal{O}^{k+1}\right)=V\left(\Psi^{k}, \mathcal{O}^{k}\right)-\beta\left|F\left(\Psi^{k+1 / 2}, \mathcal{O}^{k+1 / 2}\right)\right|^{2} .
$$

That is, we have the discrete-time analogue of (1.8). Similarly, for (4.2) we select $\epsilon^{k}$ on $[k h,(k+1) h)$ such that for $\mathcal{O}^{k+1 / 2}=S\left(\frac{h}{2}\right) \mathcal{O}^{k}$

$$
\epsilon^{k}=F\left(\Psi^{k+1 / 2}, \mathcal{O}^{k+1 / 2}\right), \quad \Psi^{k+1 / 2}=\frac{\hat{\Psi}^{k+1}+\hat{\Psi}^{k}}{2} .
$$

Copyright (c) by SIAM. Unauthorized reproduction of this article is prohibited. 
Then $\Psi^{k}$ satisfies the closed loop system

$$
\begin{aligned}
& \frac{\hat{\Psi}^{k+1}-\hat{\Psi}^{k}}{h}=\epsilon^{k} B \frac{\hat{\Psi}^{k+1}+\hat{\Psi}^{k}}{2}, \quad \hat{\Psi}^{k}=S\left(\frac{h}{2}\right) \Psi^{k}, \\
& \epsilon^{k}=F\left(\Psi^{k+1 / 2}, \mathcal{O}^{k+1 / 2}\right), \quad \Psi^{k+1}=S\left(\frac{h}{2}\right) \hat{\Psi}^{k+1} .
\end{aligned}
$$

Since

$$
V\left(S\left(\frac{h}{2}\right) \hat{\Psi}^{k+1}, S\left(\frac{h}{2}\right) \mathcal{O}^{k+\frac{1}{2}}\right)=V\left(\hat{\Psi}^{k+1}, \mathcal{O}^{k+\frac{1}{2}}\right),
$$

(4.5) holds for the closed loop (4.7).

Finally we define the nonlinear operator $\mathcal{A}(t)$ by

$$
\mathcal{A}(t) \Psi=A+F(\Psi, \mathcal{O}(t)) B \Psi, \quad \Psi \in \operatorname{dom}(A),
$$

for given $\mathcal{O} \in C(0, T ; X)$. Since $F$ is Lipschitz in $\Psi \in X$, the same proof as above provides that if $\Psi^{k}$ is the solution to the discrete-time closed loop system (4.4) and $\Psi$ satisfies the closed loop system (2.4), then

$$
\left|\Psi_{h}(t)-\Psi(t)\right|_{X} \rightarrow 0 \text { uniformly in } t \in[0, T]
$$

for both methods.

\section{Asymptotic tracking.}

5.1. Discrete spectral case. The objective of this section is to analyze the asymptotic properties of the controlled system (1.1) for the functionals $V_{1}$ and $V_{2}$. Unless specified otherwise, we assume in the context of functional $V_{1}$ that $\mathcal{O}$ appearing in (1.4) is of the form

$$
\mathcal{O}(t)=e^{-i\left(\lambda_{k_{0}} t-\hat{\theta}\right)} \psi_{k_{0}}
$$

for some eigenpair $\left(\lambda_{k_{0}}, \psi_{k_{0}}\right)$ of $\mathcal{H}_{0}$ and phase $\hat{\theta}$. For $V_{2}$ we choose

$$
\mathcal{O}=e^{i \hat{\theta}} \psi_{k_{0}} .
$$

We assume that

$$
\left\{e^{i\left(\lambda_{k}-\lambda_{k_{0}}\right) \tau}\right\}_{k=1}^{\infty} \cup\left\{e^{-i\left(\lambda_{k}-\lambda_{k_{0}}\right) \tau}\right\}_{k=1, k \neq k_{0}}^{\infty} \text { is } \omega \text {-independent in } L^{2}(0, T)
$$

for some $T>0$ and that

$$
\mu_{k_{0}}^{k}=\left(\psi_{k_{0}}, \mu \psi_{k}\right)_{X} \neq 0 \text { for all } k=1,2, \ldots
$$

A sequence $\left\{\varphi_{k}\right\}_{k=-\infty}^{\infty}$ is called $\omega$-independent if $\sum_{k=-\infty}^{\infty} c_{k} \varphi_{k}=0$ implies that $c_{k}=0$ for all $k[\mathrm{Y}]$. It is further assumed that

$$
\left\{S(t) \Psi_{0}, t \geq 0\right\} \text { is relatively compact in } X,
$$

and

$$
\text { then } \operatorname{Re}\left(\Psi_{0}, \mathcal{O}(0)\right)_{X} \neq-1 \text {. }
$$


Let us briefly comment on assumptions (5.1) and (5.3). Assumption (5.3) holds, for example, if $\operatorname{dom}\left(\mathcal{H}_{0}\right)$ is compact in $H$ and $\Psi_{0} \in V \times V$. In case $\Omega$ is unbounded we may assume that $W=V \times L^{p}(\Omega), p>2$, is compactly embedded in $H=L^{2}(\Omega)$. Then, if $\Psi_{0} \in W \times W$ and $S(t)$ leaves $W \times W$ invariant [IK1], we have (5.3).

The following lemma addresses condition (5.1).

Lemma 5.1. If there exits a constant $\delta>0$ such that $\left|\lambda_{k}+\lambda_{\ell}-2 \lambda_{k_{0}}\right| \geq \delta$ for all $k, \ell \geq 1$ with $\ell \neq k_{0}$, and $\left|\lambda_{k}-\lambda_{\ell}\right| \geq \delta$ for all $k \neq \ell$, then $\left\{e^{i\left(\lambda_{k}-\lambda_{k_{0}}\right) \tau}\right\}_{k=1}^{\infty} \cup$ $\left\{e^{-i\left(\lambda_{k}-\lambda_{k_{0}}\right) \tau}\right\}_{k=1, k \neq k_{0}}^{\infty}$ is $\omega$-independent for sufficiently large $T>0$.

We shall refer to

$$
\left|\lambda_{k}+\lambda_{\ell}-2 \lambda_{k_{0}}\right| \geq \delta>0 \text { for all } k, \ell \geq 1 \text { with } \ell \neq k_{0}
$$

as the gap condition. It is satisfied, for example, if $\psi_{k_{0}}$ is the ground state, and the associated eigenvalue $\lambda_{1}$ is not an accumulation point of the spectrum. It also holds true for arbitrary choice of $k_{0}$ if $\lambda_{k}$ behave like $k^{2}$ which, for example, is the case of the one-dimensional potential box or the case of the harmonic oscillator [C].

Proof. Let $\left\{\mu_{\ell}\right\}_{\ell \in I}$ be a real number sequence defined by

$$
\mu_{k}=\lambda_{k}-\lambda_{k_{0}}, \quad k \geq 1, \quad \mu_{-k}=-\left(\lambda_{k}-\lambda_{k_{0}}\right), \quad k \neq k_{0},
$$

where $I=\mathbb{Z} \backslash\left\{0, k_{0}\right\}$. It follows from the assumption that

$$
\left|\mu_{m}-\mu_{\ell}\right| \geq \delta, \quad m \neq \ell .
$$

From Ingham's theorem [I], if $T>\frac{2 \pi}{\delta}$, there exits a positive constant $c$, depending on $T$ and $\delta>0$, such that

$$
c \sum_{m \in I}\left|a_{m}\right|^{2} \leq\left.\int_{0}^{T} f(\tau)\right|^{2} d \tau
$$

for

$$
f(\tau)=\sum_{m \in I} a_{m} e^{i \mu_{m} \tau}
$$

From (1.10) and (1.13) we have

$$
V(t)-V(0)=-\beta \int_{0}^{t}|\epsilon(s)|^{2} d s \text { for all } t>0,
$$

where $V(t)=V_{i}(\Psi(t), \mathcal{O}(t))$ with $i=1$ or $i=2$. Since $V(t) \geq 0$ we have $\int_{0}^{\infty}|\epsilon|^{2} d s<$ $\infty$, and

$$
\lim _{t \rightarrow \infty} \int_{0}^{t} S(t-s) \epsilon(s) B \Psi(s) d s \text { exists. }
$$

It follows that $\left\{\int_{0}^{t} S(t-s) \epsilon(s) B \Psi(s): t \geq 0\right\}$ is relatively compact. Together with (5.3) we conclude that $\{\Psi(t): t \geq 0\}$ is relatively compact. We shall proceed with the asymptotic analysis utilizing assumptions (5.1), (5.2), (5.3) and summarize the results in a theorem at the end.

Since $\{\Psi(t): t \geq 0\}$ and $\{\mathcal{O}(t): t \geq 0\}$ are relatively compact in $X$ there exists a sequence $\left\{t_{n}\right\} \rightarrow \infty$ and elements $\Psi_{\infty} \in X, \mathcal{O}_{\infty} \in X$ such that

$$
\lim _{n \rightarrow \infty} \Psi\left(t_{n}\right)=\Psi_{\infty} \quad \text { and } \quad \lim _{n \rightarrow \infty} \mathcal{O}\left(t_{n}\right)=\mathcal{O}_{\infty}
$$

Copyright $@$ by SIAM. Unauthorized reproduction of this article is prohibited. 
in particular, $\Psi_{\infty}, \mathcal{O}_{\infty}$ are in the $\omega$-limit sets of (2.2) and (2.3), respectively. Let us recall that the $\omega$-limit set $\Omega$ of an orbit $\{\Psi(t): t \geq 0\}$ is defined as

$$
\Omega=\cap_{s>0} \overline{\{\Psi(t): t \geq s\}}
$$

see, e.g., [T]. Note that for any $\tau>0$

$$
\begin{aligned}
& \left|\Psi\left(t_{n}+\tau\right)-S(\tau) \Psi_{\infty}\right|_{X} \\
& \quad \leq\left|\Psi\left(t_{n}+\tau\right)-S(\tau) \Psi\left(t_{n}\right)\right|_{X}+\left|S(\tau) \Psi\left(t_{n}\right)-S(\tau) \Psi_{\infty}\right|_{X} \\
& \quad \leq\left|\int_{0}^{\tau} S(\tau-s) \epsilon\left(t_{n}+s\right) \Psi\left(t_{n}+s\right) d s\right|+\left|\Psi\left(t_{n}\right)-\Psi_{\infty}\right| \\
& \quad \leq \int_{t_{n}}^{t_{n}+\tau}|\epsilon(s)| d s+\left|\Psi\left(t_{n}\right)-\Psi_{\infty}\right| \\
& \quad \leq \sqrt{\tau}\left(\int_{t_{n}}^{t_{n}+\tau}|\epsilon(s)|^{2} d s\right)^{\frac{1}{2}}+\left|\Psi\left(t_{n}\right)-\Psi_{\infty}\right| \rightarrow 0 \text { as } n \rightarrow \infty .
\end{aligned}
$$

Since $\epsilon \in L^{2}(0, \infty)$ it follows that $\Psi\left(t_{n}+\tau\right) \rightarrow S(\tau) \Psi_{\infty}$ and analogously $\mathcal{O}\left(t_{n}+\tau\right) \rightarrow$ $S(\tau) \mathcal{O}_{\infty}$ uniformly with respect to $\tau \in(0, \infty)$. Here $S(\tau) \Psi_{\infty}$ and $S(\tau) \mathcal{O}_{\infty}$ are the mild solutions to

$$
\begin{array}{ll}
\frac{d}{d t} \Psi_{\infty}(t)=A_{0} \Psi_{\infty}(t), & \Psi_{\infty}(0)=\Psi_{\infty} \\
\frac{d}{d t} \mathcal{O}_{\infty}(t)=A_{0} \mathcal{O}_{\infty}(t), & \mathcal{O}_{\infty}(0)=\mathcal{O}_{\infty}
\end{array}
$$

Hence they are of the form

$$
\begin{aligned}
& \Psi_{\infty}(\tau)=\sum_{k=1}^{\infty} B_{k} e^{-i\left(\lambda_{k} \tau-\theta_{k}\right)} \psi_{k}, \\
& \mathcal{O}_{\infty}(\tau)=e^{-i\left(\lambda_{k_{0}} \tau-\tilde{\theta}_{k_{o}}\right)} \psi_{k_{0}},
\end{aligned}
$$

with $0 \leq \theta_{k}, \tilde{\theta}_{k_{0}}<\pi$ and $\sum_{k=1}^{\infty}\left|B_{k}\right|^{2}=1$.

Since

$$
\epsilon\left(t_{n}+\cdot\right)=F\left(\Psi\left(t_{n}+\cdot\right), \mathcal{O}\left(t_{n}+\cdot\right)\right) \rightarrow 0 \text { in } L^{2}(0, \infty), \text { as } t_{n} \rightarrow \infty,
$$

by Lipschitz continuity of $(\Psi, \mathcal{O}) \in X \times X \rightarrow F(\Psi, \mathcal{O}) \in \mathbb{R}$ and Lebesgue's bounded convergence theorem, we have

$$
F\left(\Psi_{\infty}(\tau), \mathcal{O}_{\infty}(\tau)\right)=0 \text { for } \tau \geq 0
$$

It follows now that

$$
\begin{aligned}
& F_{1}\left(\Psi_{\infty}(\tau), \mathcal{O}_{\infty}(\tau)\right)=-\frac{1}{\beta} \operatorname{Im}\left(\sum_{k=1}^{\infty} B_{k} e^{i\left(\left(\lambda_{k}-\lambda_{k_{0}}\right) \tau-\theta_{k}+\tilde{\theta}_{k_{0}}\right)} \mu_{k_{0}}^{k}\right) \\
& =-\frac{1}{\beta} \sum_{k=1}^{\infty} \mu_{k_{0}}^{k} B_{k}\left(\cos \left(\theta_{k}-\tilde{\theta}_{k_{0}}\right) \sin \left(\left(\lambda_{k}-\lambda_{k_{0}}\right) \tau\right)-\sin \left(\theta_{k}-\tilde{\theta}_{k_{0}}\right) \cos \left(\left(\lambda_{k}-\lambda_{k_{0}}\right) \tau\right)\right)=0,
\end{aligned}
$$

where $\mu_{k_{0}}^{k}=\left(\psi_{k_{0}}, \mu \psi_{k}\right)_{X}$.

Copyright (c) by SIAM. Unauthorized reproduction of this article is prohibited. 
By (5.1) the set $\left\{\cos \left(\left(\lambda_{k}-\lambda_{k_{0}}\right) \tau\right), \sin \left(\left(\lambda_{k}-\lambda_{k_{0}}\right) \tau\right)\right\}$ is $\omega$-independent in $L^{2}(0, T)$. From (5.10) it thus follows, using (5.2), that $B_{k}=0$ for all $k \neq k_{0}$. Moreover, since $\left|\Psi_{\infty}\right|=1$, we have $\theta_{k_{0}}=\tilde{\theta}_{k_{0}}$ and $B_{k_{0}}=1$.

Here the case $B_{k_{0}}=-1$ can be excluded since it implies that

$$
V_{1}\left(\Psi_{\infty}(\tau), \mathcal{O}_{\infty}(\tau)\right)=1+\operatorname{Re}\left(e^{-i\left(\lambda_{k_{0}} \tau-\theta_{k_{0}}\right)} \psi_{k_{0}}, e^{-i\left(\lambda_{k_{0}} \tau-\theta_{k_{0}}\right)} \psi_{k_{0}}\right)_{X}=2 .
$$

But by (5.4) we have

$$
V_{1}\left(\Psi_{0}, \mathcal{O}(0)\right)=1-\operatorname{Re}\left(e^{i \hat{\theta}} \psi_{k_{0}}, \Psi_{0}\right)_{X} \leq 2 .
$$

Moreover $V_{1}(\Psi(t), \mathcal{O}(t))$ decays along the trajectory, i.e., $\frac{d}{d t} V_{1}(\Psi(t), \mathcal{O}(t)) \leq 0$, which is in contradiction to (5.11) and (5.12), and hence $B_{k_{0}}=-1$ cannot occur.

Since the $\omega$-limit pair $\left(\Psi_{\infty}, \mathcal{O}_{\infty}\right)$ was arbitrary, it follows from the Lyapunov functionals (1.4) that $\lim _{t \rightarrow \infty} V_{1}(\Psi(t), \mathcal{O}(t))=0$, i.e., $\Psi(t)$ asymptotically approaches the orbit $\mathcal{O}(t)$.

Similarly,

$$
\begin{gathered}
F_{2}\left(\Psi_{\infty}(\tau), \mathcal{O}_{\infty}\right)=-\frac{1}{\beta} \operatorname{Im}\left(B_{k_{0}} \sum_{k \neq k_{0}} B_{k} e^{i\left(\left(\lambda_{k}-\lambda_{k_{0}}\right) \tau+\theta_{k_{0}}-\theta_{k}\right)} \mu_{k_{0}}^{k}\right) \\
=-\frac{1}{\beta} \sum_{k \neq k_{0}} \mu_{k_{0}}^{k} B_{k_{0}} B_{k}\left(\cos \left(\theta_{k_{0}}-\theta_{k}\right) \sin \left(\left(\lambda_{k}-\lambda_{k_{0}}\right) \tau\right)\right. \\
\left.+\sin \left(\theta_{k_{0}}-\theta_{k}\right) \cos \left(\left(\lambda_{k}-\lambda_{k_{0}}\right) \tau\right)\right)=0 .
\end{gathered}
$$

In case $\left(\Psi_{0}, \psi_{k_{0}}\right)_{X} \neq 0$ we have $B_{k_{0}} \neq 0$. In fact, $\left(\Psi_{0}, \psi_{k_{0}}\right)_{X} \neq 0$ implies that $V_{2}\left(\Psi_{0}, \mathcal{O}(0)\right)=\frac{1}{2}\left(1-\left|\left(\mathcal{O}(0), \Psi_{0}\right)_{X}\right|^{2}\right)<\frac{1}{2}$, and, since $\frac{d}{d t} V_{2} \leq 0$, we have $V_{2}\left(\Psi_{\infty}, \mathcal{O}_{\infty}\right) \leq \frac{1}{2}$. If $B_{k_{0}}=0$, then $(\Psi(\infty), \mathcal{O}(\infty))_{X}=0$ and $V_{2}(\Psi(\infty), \mathcal{O}(\infty))=\frac{1}{2}$, which gives a contradiction.

Thus, with (5.1) and (5.2) holding, it follows that for $F_{2}$ given in (5.13) $B_{k}=0$ for $k \neq k_{0}$ and thus $B_{k_{0}}= \pm 1$. Since the element in the $\omega$-limit set was arbitrary we conclude that $V_{2}(\Psi(t), \mathcal{O}(t)) \rightarrow 0$ as $t \rightarrow \infty$, which means that the trajectory $\Psi(t)$ approaches the manifold $\left\{e^{i \theta} \psi_{k_{0}}: \theta \in[0,2 \pi)\right\}$ as $t \rightarrow \infty$.

We summarize the above discussion as a theorem.

Theorem 5.2. Assume that (5.1), (5.2), and (5.3) hold.

(a) If, in addition, (5.4) is satisfied, then $\lim _{t \rightarrow \infty} V_{1}(\Psi(t), \mathcal{O}(t))=0$ for the feedback law given by $F_{1}$.

(b) If, in addition, $\left(\Psi_{0}, \psi_{k_{0}}\right)_{X} \neq 0$, then $\lim _{t \rightarrow \infty} V_{2}(\Psi(t), \mathcal{O}(t))=0$ for the feedback law given by $F_{2}$.

Remark 5.1. For the harmonic oscillator case we have

$$
\mathcal{H}_{0} \psi=-\frac{d^{2}}{d x^{2}} \psi+x^{2} \psi, \quad x \in \mathbb{R}=\Omega .
$$

Then the eigenpairs $\left\{\left(\lambda_{k}, \psi_{k}\right)\right\}_{k=1}^{\infty}$ are given by

$$
\lambda_{k}=2 k-1, \quad \psi_{k}(x)=\hat{c} H_{k-1}(x) e^{-\frac{x^{2}}{2}},
$$

where $H_{k}$ is the Hermite polynomial of degree $k$ and $\hat{c}$ is a normalizing factor. In this case we have

$$
\lambda_{k_{0}-\ell}-\lambda_{k_{0}}=-\left(\lambda_{k_{0}+\ell}-\lambda_{k_{0}}\right), \quad 1 \leq \ell \leq k_{0}-1,
$$


and the gap condition $\left|\lambda_{k}+\lambda_{\ell}-2 \lambda_{k_{0}}\right|>\delta$ is not satisfied. Thus, $\int_{0}^{T}\left|F_{1}(\Psi(\tau), \mathcal{O}(\tau))\right|^{2} d \tau$ $=0$ implies

$$
\operatorname{Im}\left(B_{k_{0}+\ell} e^{i\left(\lambda_{\ell} \tau-\theta_{k_{0}+\ell}+\tilde{\theta}_{k_{0}}\right)} \mu_{k_{0}}^{k_{0}+\ell}+B_{k_{0}-\ell} e^{-i\left(\lambda_{\ell} \tau-\theta_{k_{0}-\ell}+\tilde{\theta}_{k_{0}}\right)} \mu_{k_{0}}^{k_{0}-\ell}\right)=0
$$

for $1 \leq \ell<k_{0}$. That is, $B_{k_{0}+\ell}$ and $B_{k_{0}-\ell}$ are not necessarily zero and thus $\Psi_{\infty}(\tau)$ is distributed over energy levels $1 \leq \ell \leq 2 k_{0}-1$.

We now turn to the case when the gap condition $\left|\lambda_{k}+\lambda_{\ell}-2 \lambda_{k_{0}}\right|>\delta$ is violated. Then more than one control operator $\mu$ is required to guarantee the tracking property of our feedback law and we consider (1.14).

Then for $V_{1}(\Psi, \mathcal{O})=1-\operatorname{Re}(\mathcal{O}, \Psi)_{X}$ we find

$$
\frac{d}{d t} V_{1}(\Psi(t), \mathcal{O}(t))=\sum_{j=1}^{m} \epsilon_{j} \operatorname{Im}\left(\mathcal{O}(t), \mu_{j} \Psi(t)\right)_{X}
$$

which suggests feedback laws of the form

$$
\epsilon_{j}(t)=F_{1, j}(\Psi(t), \mathcal{O}(t))=-\frac{1}{\beta} \operatorname{Im}\left(\mathcal{O}(t), \mu_{j} \Psi(t)\right) .
$$

For the cost functional $V_{2}$ we obtain the feedback laws

$$
\epsilon_{j}(t)=F_{2, j}(\Psi(t), \mathcal{O})=-\frac{1}{\beta} \operatorname{Im}\left(\overline{(\mathcal{O}, \Psi(t))}\left(\mathcal{O}, \mu_{j} \Psi(t)\right)\right)
$$

for $j=1, \ldots, m$. As before we obtain, for either of the two cost functionals,

$$
V(t)-V(0)=-\beta \int_{0}^{t} \sum_{j=1}^{m}\left|\epsilon_{j}(s)\right|^{2} d s,
$$

and hence $\epsilon_{j} \in L^{2}(0, \infty)$ for each $j=1, \ldots, m$.

In the following discussion we assume (5.3), i.e., that $\left\{S(t) \Psi_{0}: t \geq 0\right\}$ is relatively compact. Then $\{\Psi(t): t \geq 0\}$ is relatively compact as well and, proceeding as at the beginning of this section, we obtain for each $j=1, \ldots, m$

$$
F_{1, j}\left(\Psi_{\infty}(\tau), \mathcal{O}_{\infty}(\tau)\right)=-\frac{1}{\beta} \operatorname{Im}\left(\mathcal{O}_{\infty}(\tau), \mu_{j} \Psi_{\infty}(\tau)\right)=0 \text { for } \tau \geq 0, j=1, \ldots, m,
$$

in particular

$$
\operatorname{Im}\left(\sum_{k=1}^{\infty} B_{k} e^{i\left(\left(\lambda_{k}-\lambda_{k_{0}}\right) \tau-\theta_{k}+\tilde{\theta}_{k_{0}}\right)}\left(\mu_{j}\right)_{k_{0}}^{k}\right)=0 \text { for } j=1, \ldots, m
$$

where

$$
\left(\mu_{j}\right)_{k_{0}}^{k}=\left(\psi_{k_{0}}, \mu_{j} \psi_{k}\right)_{X} .
$$

We henceforth consider the case $m=2$. Suppose that $\lambda_{\bar{k}}+\lambda_{\bar{\ell}}-2 \lambda_{k_{0}}=0$ for a single pair $(\bar{k}, \bar{\ell}), \bar{\ell} \neq k_{0}$, and that otherwise (5.1) holds. Then $\lambda_{\bar{k}}-\lambda_{k_{0}}=-\left(\lambda_{\bar{\ell}}-\lambda_{k_{0}}\right)$ and for the feedback law associated to $V_{1}$ we have

$$
\operatorname{Im}\left(B_{\bar{k}} e^{i\left(\left(\lambda_{\bar{k}}-\lambda_{k_{0}}\right) \tau-\theta_{\bar{k}}+\tilde{\theta}_{k_{0}}\right)}\left(\mu_{j}\right)_{k_{0}}^{\bar{k}}+B_{\bar{\ell}} e^{i\left(-\left(\lambda_{\bar{k}}-\lambda_{k_{0}}\right) \tau-\theta_{\bar{\ell}}+\tilde{\theta}_{k_{0}}\right)}\left(\mu_{j}\right)_{k_{0}}^{\bar{\ell}}\right)=0
$$

Copyright $@$ by SIAM. Unauthorized reproduction of this article is prohibited. 
for $j=1,2$. If

$$
\operatorname{rank}\left(\begin{array}{cc}
\left(\mu_{1}\right)_{k_{0}}^{\bar{k}} & \left(\mu_{1}\right)_{k_{0}}^{\bar{\ell}} \\
\left(\mu_{2}\right)_{k_{0}}^{\bar{k}} & \left(\mu_{2}\right)_{k_{0}}^{\bar{\ell}}
\end{array}\right)=2,
$$

then from (5.16), it follows that $B_{\bar{k}}=B_{\bar{\ell}}=0$. If moreover

$$
\text { for each } k \text { there exists } j \in\{1,2\} \text { such that }\left(\mu_{j}\right)_{k_{0}}^{k} \neq 0 \text {, }
$$

then $B_{k}=0$ for all $k \neq k_{0}, B_{k_{0}}=1$, and $\theta_{k_{0}}=\tilde{\theta}_{k_{0}}$. As a consequence we have $\lim _{t \rightarrow \infty} V_{1}(\Psi(t), \mathcal{O}(t))=0$.

In general let

$$
\lambda_{k_{i}}+\lambda_{\ell_{i}}-2 \lambda_{k_{0}}=0
$$

for multiple pairs $\left(k_{i}, \ell_{i}\right)$ with $\ell_{i} \neq k_{0}$. If we assume that (5.18) holds and

$$
\operatorname{rank}\left(\begin{array}{cc}
\left(\mu_{1}\right)_{k_{0}}^{k_{i}} & \left(\mu_{1}\right)_{k_{0}}^{\ell_{i}} \\
\left(\mu_{2}\right)_{k_{0}}^{k_{i}} & \left(\mu_{2}\right)_{k_{0}}^{\ell_{i}}
\end{array}\right)=2 \text { for each }\left(k_{i}, \ell_{i}\right) \text { pair }
$$

then $B_{k_{i}}=B_{\ell_{i}}=0$, and in particular $B_{k}=0$ for all $k$. Again $\lim _{t \rightarrow \infty} V_{1}(\Psi(t), \mathcal{O}(t))=$ 0 follows.

Turning to the feedback law corresponding to $V_{2}$ we find

$$
F_{2, j}\left(\Psi_{\infty}(\tau), \mathcal{O}_{\infty}\right)=-\frac{1}{\beta} \operatorname{Im}\left(\overline{\left(\mathcal{O}_{\infty}, \Psi_{\infty}(\tau)\right)_{X}}\left(\mathcal{O}_{\infty}, \mu_{j} \Psi_{\infty}(\tau)\right)\right)=0
$$

for $\tau \geq 0, j=1, \ldots, m$. Consequently

$$
B_{k_{0}} \operatorname{Im}\left(B_{k} e^{i\left(\left(\lambda_{k}-\lambda_{k_{0}}\right) \tau+\theta_{k_{0}}-\theta_{k}\right)}\left(\mu_{j}\right)_{k_{0}}^{k}+B_{\ell} e^{i\left(-\left(\lambda_{k}-\lambda_{k_{0}}\right) \tau+\theta_{k_{0}}-\theta_{\ell}\right)}\left(\mu_{j}\right)_{k_{0}}^{\ell}\right)=0 .
$$

In case $\lambda_{k_{i}}+\lambda_{\ell_{i}}-2 k_{0}=0$ for multiple pairs $\left(k_{i}, \ell_{i}\right)$ with $\ell_{i} \neq k_{0}$, then (5.18), (5.19), and $B_{k_{0}} \neq 0$ (which is implied by $\left(\Psi_{0}, \psi_{k_{0}}\right)_{X} \neq 0$ ) imply that $B_{k_{i}}=B_{\ell_{i}}=0$ and in particular $B_{k}=0$ for all $k \neq k_{0}$. Again we can draw the same conclusion as in Theorem 5.2.

We can conclude that even in the degenerate case when the gap condition is violated, only two independent moments are sufficient to guarantee the asymptotic tracking properties of $V_{i}(\Psi(t), \mathcal{O}(t)), i=1,2$. More precisely we have the following result.

THEOREM 5.3. Consider control potentials of the form $\tilde{\mu}(t)=\sum_{j=1}^{2} \epsilon_{j}(t) \mu_{j}$ with $\mu_{j} \in \mathcal{L}(H)$. Assume that (5.1) holds except for finitely many pairs $\left(k_{i}, \ell_{i}\right), \ell_{i} \neq k_{0}$, and that (5.18) and (5.19), as well as (5.3), are satisfied.

(a) If, in addition, (5.4) holds, then $\lim _{t \rightarrow \infty} V_{1}(\Psi(t), \mathcal{O}(t))=0$ for the feedback law given by $F_{1}$.

(b) If, in addition, $\left(\Psi_{0}, \psi_{k_{0}}\right)_{X} \neq 0$, then $\lim _{t \rightarrow \infty} V_{2}(\Psi(t), \mathcal{O}(t))=0$ for the feedback law given by $F_{2}$.

Copyright (c) by SIAM. Unauthorized reproduction of this article is prohibited. 
5.2. Continuous spectral case. In this subsection we assume that the positive, self-adjoint operator $\mathcal{H}_{0}$ has a spectral resolution of the form

$$
\mathcal{H}_{0} \psi=\lambda_{0}\left(\psi, \psi_{0}\right) \psi_{0}+\int_{\lambda_{1}}^{\infty} \lambda E(\lambda) \psi d \lambda \text { for } \psi \in \operatorname{dom}\left(\mathcal{H}_{0}\right)
$$

where $0<\lambda_{0}<\lambda_{1}$, and $E(\lambda)$ is a family of projections with $\int_{\lambda_{1}}^{\infty}|\lambda E(\lambda) \psi|_{X}^{2} d \lambda<\infty$ for all $\psi \in \operatorname{dom}\left(\mathcal{H}_{0}\right)$ and $E \in L_{\text {loc }}^{2}\left(\lambda_{1}, \infty ; \mathcal{L}(X)\right)$; see, e.g., [Yo, pp. 352]. and

Proceeding as in section 5.1 and assuming (5.3), we find that $\mathcal{O}_{\infty}(\tau)=e^{-i\left(\lambda_{0} \tau-\tilde{\theta}_{0}\right)} \psi_{0}$

$$
\Psi_{\infty}(\tau)=B_{0} e^{-i\left(\lambda_{0} \tau-\theta_{0}\right)} \psi_{0}+\int_{\lambda_{1}}^{\infty} e^{-i(\lambda \tau-\theta(\lambda))} E(\lambda) \Psi_{\infty} d \lambda,
$$

where $\Psi_{\infty}$, with $\left|\Psi_{\infty}\right|_{X}=1$, is in the $\omega$-limit set of $\Psi(t)$. We obtain

$$
\begin{aligned}
& \epsilon_{\infty}(\tau)=F_{1}\left(\psi_{\infty}(\tau), \mathcal{O}_{\infty}(\tau)\right)=-\frac{1}{\beta} \operatorname{Im}\left(\mathcal{O}_{\infty}, \mu \Psi_{\infty}\right)_{X} \\
& =-\frac{1}{\beta} B_{0} \operatorname{Im}\left(e^{i\left(\tilde{\theta}_{0}-\theta_{0}\right)}\left(\psi_{0}, \mu \psi_{0}\right)_{X}\right)-\frac{1}{\beta} \operatorname{Im} \int_{\lambda_{1}}^{\infty} e^{i\left(\left(\lambda-\lambda_{0}\right) \tau+\tilde{\theta}_{0}-\theta(\lambda)\right)}\left(\psi_{0}, \mu E(\lambda) \Psi_{\infty}\right)_{X} \\
& =-\frac{1}{\beta} B_{0} \sin \left(\tilde{\theta}_{0}-\theta_{0}\right)\left(\psi_{0}, \mu \psi_{0}\right)_{X}-\frac{1}{\beta} \operatorname{Im}\left(\int_{\lambda_{1}}^{\infty} e^{i\left(\left(\lambda-\lambda_{0}\right) \tau+\tilde{\theta}_{0}-\theta(\lambda)\right)} \tilde{B}(\lambda)\right),
\end{aligned}
$$

where $\tilde{B}(\lambda)=\left(\psi_{0}, \mu E(\lambda) \Psi_{\infty}\right)$. Equivalently this can be expressed as

$$
\begin{aligned}
& \epsilon_{\infty}(\tau)=-\frac{1}{\beta} B_{0} \sin \left(\tilde{\theta}_{0}-\theta_{0}\right)\left(\psi_{0}, \mu \psi_{0}\right)_{X} \\
& -\frac{1}{\beta}\left(\int_{\lambda_{1}}^{\infty} \tilde{B}(\lambda)\left[\cos \left(\theta(\lambda)-\tilde{\theta}_{0}\right) \sin \left(\left(\lambda-\lambda_{0}\right) \tau\right)-\sin \left(\theta(\lambda)-\tilde{\theta}_{0}\right) \cos \left(\left(\lambda-\lambda_{0}\right) \tau\right)\right]\right) .
\end{aligned}
$$

Hence by the Fourier-Plancherel theorem

$$
\int_{0}^{\infty}\left|\epsilon_{\infty}(\tau)\right|^{2} d \tau=\left|B_{0} \sin \left(\theta_{0}-\tilde{\theta}_{0}\right)\left(\psi_{0}, \mu \psi_{0}\right)_{X}\right|^{2}+\int_{\lambda_{1}}^{\infty}|\tilde{B}(\lambda)|^{2} d \lambda=0,
$$

and thus

$$
\tilde{B}(\lambda)=0, \quad B_{0} \sin \left(\theta_{0}-\tilde{\theta}_{0}\right)\left(\psi_{0}, \mu \psi_{0}\right)=0
$$

Assuming that

$$
\left(\psi_{0}, \mu \psi_{0}\right) \neq 0 \text {, and that } \tilde{B}(\cdot)=0 \text { implies } E(\cdot) \Psi_{\infty}(\cdot)=0 \text { for every } \Psi_{\infty},
$$

we have $\theta_{0}=\tilde{\theta}_{0}$ and $E \Psi_{\infty}=0$. Since $\left|\Psi_{\infty}\right|=1$ we have $B_{0}=1$, and therefore $V_{1}(\Psi(t), \mathcal{O}(t))=1-\operatorname{Re}(\mathcal{O}(t), \Psi(t)) \rightarrow 0$ as $t \rightarrow \infty$.

5.3. General target. In this subsection we consider the case when the orbit that is tracked by means of $V_{1}$ is chosen based on multiple eigenstates according to

$$
\mathcal{O}(t)=\sum_{\ell=1}^{M} \hat{\alpha}_{\ell} e^{-i\left(\lambda_{\ell} t-\hat{\vartheta}_{\ell}\right)} \psi_{\ell},
$$

Copyright (C) by SIAM. Unauthorized reproduction of this article is prohibited. 
with $\sum_{\ell=1}^{M}\left|\hat{\alpha}_{\ell}\right|^{2}=1, \hat{\alpha}_{\ell} \neq 0$, phases $\hat{\vartheta}_{\ell} \in[0,2 \pi)$, for all $\ell$, and $M \geq 2$. Throughout this section we consider the point-spectrum situation of section 5.1 with simple eigenvalues.

Analogous to section 5.1 we assume that (5.3) holds, and we replace (5.1) by

$$
\bigcup_{\ell=1}^{M}\left(\left\{e^{i\left(\lambda_{k}-\lambda_{\ell}\right) \tau}\right\}_{k=1}^{\infty} \cup\left\{e^{-i\left(\lambda_{k}-\lambda_{\ell}\right) \tau}\right\}_{k=1, k \neq \ell}^{\infty}\right) \text { is } \omega \text {-independent in } L^{2}(0, T)
$$

for some $T>0$, and we replace (5.2) by

$$
\mu_{\ell}^{k}=\left(\psi_{\ell}, \mu \psi_{k}\right)_{X} \neq 0 \text { for all } \ell=1, \ldots, M, k=1,2, \ldots
$$

In (5.22) the union $\dot{U}$ is defined such that multiple occurrences of the value $\lambda_{k}-\lambda_{\ell}$ are omitted. Note that (5.21) holds if a real sequence $\dot{\bigcup}_{\ell=1}^{M}\left(\left\{\lambda_{k}-\lambda_{\ell}\right\}_{k=1}^{\infty} \cup\left\{-\left(\lambda_{k}-\right.\right.\right.$ $\left.\left.\left.\lambda_{\ell}\right)\right\}_{k=1, k \neq \ell}^{\infty}\right)$ satisfies the uniform gap condition as in Lemma 5.1.

As in section 5.1, we consider the feedback control

$$
\epsilon(t)=-\frac{1}{\beta} \operatorname{Im}(\mathcal{O}(t), \mu \Psi(t))_{X}=F_{1}(\Psi(t), \mathcal{O}(t))
$$

and find that there exist $0 \leq \tilde{\theta}_{\ell}, \theta_{k}<\pi$ and $B_{k}$, with $\sum_{k=1}^{\infty} B_{k}^{2}=1$, such that

$$
\mathcal{O}_{\infty}(\tau)=\sum_{\ell=1}^{M} \alpha_{k} e^{-i\left(\lambda_{\ell} \tau-\tilde{\theta}_{\ell}\right)} \psi_{\ell}, \quad \Psi_{\infty}(\tau)=\sum_{k=1}^{\infty} B_{k} e^{-i\left(\lambda_{k} \tau-\theta_{k}\right)} \psi_{k}
$$

for $\tau \geq 0$. It follows that

$$
F_{1}\left(\Psi_{\infty}(\tau), \mathcal{O}_{\infty}(\tau)\right)=0
$$

where

$$
\begin{aligned}
& F_{1}\left(\Psi_{\infty}(\tau), \mathcal{O}_{\infty}(\tau)\right)=-\frac{1}{\beta} \operatorname{Im}\left(\mathcal{O}_{\infty}(\tau), \mu \Psi_{\infty}(\tau)\right)_{X} \\
& =-\frac{1}{\beta} \operatorname{Im}\left(\sum_{k=1}^{\infty} B_{k} \sum_{\ell=1}^{M} \alpha_{\ell} e^{i\left(\left(\lambda_{k}-\lambda_{\ell}\right) \tau-\theta_{k}+\tilde{\theta}_{\ell}\right)} \mu_{\ell}^{k}\right) \\
& =-\frac{1}{\beta} \sum_{k=1}^{\infty} B_{k} \sum_{\ell=1}^{M} \alpha_{\ell} \mu_{\ell}^{k}\left(\cos \left(\theta_{k}-\tilde{\theta}_{\ell}\right) \sin \left(\left(\lambda_{k}-\lambda_{\ell}\right) \tau\right)-\sin \left(\theta_{k}-\tilde{\theta}_{\ell}\right) \cos \left(\left(\lambda_{k}-\lambda_{\ell}\right) \tau\right)\right) .
\end{aligned}
$$

In the following discussion we argue that $\Psi_{\infty}=\mathcal{O}_{\infty}$, which implies the tracking property of the feedback control law $\epsilon$.

Conditions (5.21), (5.22) imply that

$$
\begin{aligned}
& \cos \left(\theta_{k}-\tilde{\theta}_{\ell}\right) B_{k} \alpha_{\ell}-\cos \left(\theta_{\ell}-\tilde{\theta}_{k}\right) B_{\ell} \alpha_{k}=0 \\
& \sin \left(\theta_{k}-\tilde{\theta}_{\ell}\right) B_{k} \alpha_{\ell}+\sin \left(\theta_{\ell}-\tilde{\theta}_{k}\right) B_{\ell} \alpha_{k}=0 \\
& \sum_{i=1}^{M} \sin \left(\theta_{i}-\tilde{\theta}_{i}\right) B_{i} \alpha_{i} \mu_{i}^{i}=0
\end{aligned}
$$

for $k \neq \ell, 1 \leq k, \ell \leq M$, and $B_{k}=0$ for $k>M$. 
Turning to the case $k \leq M$ note that

$$
\operatorname{det}\left(\begin{array}{cc}
\cos \left(\theta_{k}-\tilde{\theta}_{\ell}\right) & -\cos \left(\theta_{\ell}-\tilde{\theta}_{k}\right) \\
\sin \left(\theta_{k}-\tilde{\theta}_{\ell}\right) & \sin \left(\theta_{\ell}-\tilde{\theta}_{k}\right)
\end{array}\right)=\sin \left(\theta_{k}-\tilde{\theta}_{k}+\theta_{\ell}-\tilde{\theta}_{\ell}\right) .
$$

If $\sin \left(\theta_{k}-\tilde{\theta}_{k}+\theta_{\ell}-\tilde{\theta}_{\ell}\right) \neq 0$, then $B_{k}=B_{\ell}=0$ and in particular

$$
B_{k} \alpha_{\ell}=B_{\ell} \alpha_{k} \text {. }
$$

If $\sin \left(\theta_{k}-\tilde{\theta}_{k}+\theta_{\ell}-\tilde{\theta}_{\ell}\right)=0$, then for $k \neq \ell, 1 \leq \ell, k \leq M$

$$
B_{k} \alpha_{\ell}=B_{\ell} \alpha_{k}, \quad \theta_{k}-\tilde{\theta}_{\ell}+\theta_{\ell}-\tilde{\theta}_{k}=0,
$$

or

$$
B_{k} \alpha_{\ell}=-B_{\ell} \alpha_{k}, \quad \theta_{k}-\tilde{\theta}_{\ell}+\theta_{\ell}-\tilde{\theta}_{k}= \pm \pi .
$$

If $B_{k}=0$ for some $k \in\{1, \ldots, M\}$, then $B_{k}=0$ for all $k \in\{1, \ldots, M\}$, which contradicts $\sum_{k=1}^{\infty}\left|B_{k}\right|^{2}=1$. Hence $B_{k} \neq 0$ for all $k=1, \ldots, M$ and (5.27) or (5.28) hold for all $k \neq \ell, 1 \leq \ell, k \leq M$. Consequently there exists a constant $c \neq 0$ such that

$$
\frac{B_{k}}{\alpha_{k}}= \pm c
$$

for all $k=1, \ldots, M$. Since $\sum_{k=1}^{\infty}\left|B_{k}\right|^{2}=\sum_{k=1}^{\infty}\left|\alpha_{k}\right|^{2}=1$ this implies that $B_{k}= \pm \alpha_{k}$ for $k=1, \ldots, M$. If $M \geq 3$, one argues, using $\theta_{k}-\tilde{\theta}_{k} \in(-\pi, \pi)$, that (5.28) cannot occur. Then (5.27) and $M \geq 3$ imply that

$$
\theta_{k}-\tilde{\theta}_{k}=0, \quad 1 \leq k \leq M,
$$

and

$$
B_{k}=\alpha_{k} \text { or } B_{k}=-\alpha_{k} \text { for all } k=1, \ldots, M \text {. }
$$

The case $B_{k}=-\alpha_{k}$ for all $k=1, \ldots, M$ cannot occur if (5.4) holds. In fact, we can argue similarly as in (5.11)-(5.12), since again we have $V_{1}\left(\Psi_{\infty}(\tau), \mathcal{O}(\tau)\right)=1+$ $\operatorname{Re}\left(\sum_{k=1}^{M} A_{k}^{2}\right)=2$, and $V_{1}\left(\Psi_{0}, \mathcal{O}(0)\right)=1-\operatorname{Re}\left(\mathcal{O}(0), \Psi_{0}\right)_{X}<2$ by $(5.4)$.

Thus, if (5.3), (5.4) (5.21), and (5.24) hold and $M \geq 3$, then $\Psi_{\infty}=\mathcal{O}_{\infty}$ and $V_{1}(\Psi(t), \mathcal{O}(t)) \rightarrow 0$ as $t \rightarrow \infty$.

Let us turn to the case $M=2$. The case $\sin \left(\theta_{2}-\tilde{\theta}_{2}+\theta_{1}-\tilde{\theta}_{1}\right) \neq 0$ cannot occur since then $B_{k}=0$ for all $k$. Consequently

$$
B_{1} \alpha_{2}=B_{2} \alpha_{1} \quad \text { and } \quad \theta_{2}-\tilde{\theta}_{1}+\theta_{1}-\tilde{\theta}_{2}=0
$$

or

$$
B_{1} \alpha_{2}=-B_{2} \alpha_{1} \quad \text { and } \quad \theta_{2}-\tilde{\theta}_{1}+\theta_{1}-\tilde{\theta}_{2}= \pm \pi .
$$

Either of these two cases combined with the third equation in (5.25) implies that

$$
\sin \left(\theta_{2}-\tilde{\theta}_{2}\right)\left(\alpha_{1}^{2} \mu_{1}^{1}-\alpha_{2}^{2} \mu_{2}^{2}\right)=0 .
$$

Thus if $\alpha_{2}^{2} \mu_{1}^{1}-\alpha_{1}^{2} \mu_{2}^{2} \neq 0$, then similarly to the above arguments $\theta_{i}=\tilde{\theta}_{i}, B_{i}=\alpha_{i}, i=$ 1,2 , and consequently $V_{1}(\Psi(t), \mathcal{O}(t)) \rightarrow 0$ as $t \rightarrow \infty$.

We summarize the above discussion as a theorem.

TheOREM 5.4. Assume that (5.21), (5.22), (5.3), and (5.4) hold and consider the multipole target $\mathcal{O}(t)=\sum_{\ell=1}^{M} \hat{\alpha}_{\ell} e^{-i\left(\lambda_{\ell} t-\hat{\vartheta}_{\ell}\right)} \psi_{\ell}$. In case $M=2$ let $\alpha_{2}^{2} \mu_{1}^{1}-\alpha_{1}^{2} \mu_{2}^{2} \neq 0$. Then $\lim _{t \rightarrow \infty} V_{1}(\Psi(t), \mathcal{O}(t))=0$ for the feedback law given by $F_{1}$.

We end with a remark exploiting the fact that (1.1) can be integrated backwards in time.

Copyright $@$ by SIAM. Unauthorized reproduction of this article is prohibited. 
6. Numerical tests. In this section we demonstrate the feasibility of the proposed feedback laws for orbit tracking. The test example is chosen such that the gap condition (5.5) is not satisfied. Nevertheless good tracking properties are obtained with a controller consisting of two control potentials.

We set $H=L^{2}(0,1)$ and

$$
\mathcal{H}_{0} \psi=\sum_{k=1}^{\infty} \lambda_{k}\left(\psi, \psi_{k}\right)_{H} \psi_{k},
$$

where

$$
\psi_{k}(x)=\sqrt{2} \sin (k \pi x) \quad \text { and } \quad \lambda_{k}=k \pi .
$$

The control Hamiltonians are given by

$$
\left(\mu_{i} \Psi\right)(x)=b_{i}(x) \Psi(x), \quad x \in(0,1),
$$

with $i=1,2$. For computations we truncated the expansion of $\mathcal{H}_{0}$ at $N=99$, so that

$$
S_{N}(h) \Psi_{0}=\sum_{k=1}^{N} e^{-i \lambda_{k} h}\left(\Psi_{0}, \psi_{k}\right) \psi_{k} .
$$

To integrate the control Hamiltonian term the collocation method was used in the form

$$
\left(e^{\epsilon B_{N}^{i} h} \psi\right)\left(x_{n}^{N}\right)=e^{-i \epsilon b_{i}\left(x_{n}^{N}\right) h} \psi_{k}\left(x_{n}^{N}\right),
$$

where $x_{n}^{N}=\frac{n}{N}, 1 \leq n \leq N-1$. Thus, we implemented the feedback law based on the splitting method (Lie-Trotter product) in the form

$$
\begin{aligned}
& \Psi^{k+1}=S_{N}(h) \mathcal{F}_{N} e^{\left(\epsilon_{1}^{k} B_{N}^{1}+\epsilon_{2}^{k} B_{N}^{2}\right) h} \mathcal{F}_{N}^{-1} \Psi^{k} \\
& \epsilon_{j}^{k}=F_{1}\left(\Psi^{k}, \mathcal{O}^{k}\right)=-\frac{1}{\beta} \operatorname{Im}\left(\sum_{n=1}^{N-1} b_{j}\left(x_{n}^{N}\right) \mathcal{O}\left(x_{n}^{N}\right) \Psi\left(x_{n}^{N}\right)\right),
\end{aligned}
$$

where $\mathcal{F}_{N}$ and $\mathcal{F}_{N}^{-1}$ are the discrete Fourier sine transform and its inverse transform, respectively. This is an explicit method. We implemented the implicit method as described in section 4 as well. The results are very similar with respect to the tracking speed for both methods. The numerical tests that we report on are computed with $h=0.01, \beta=500$ and

$$
b_{1}=(x-.5)+1.75(x-.5)^{2}, \quad b_{2}=2.5(x-.5)^{3}-2.5(x-.5)^{4} .
$$

These control potentials satisfy the rank condition in section 5 and are selected by minimizing the tracking time by trial-and-error tests. Figure 1 shows the tracked state (real and imaginary parts) after 50 time units compared to the desired orbit. The imaginary part of the desired state is zero at $T$ and there remains some tracking error. On the right the tracking error in terms of $V_{1}\left(\Psi^{k}, \mathcal{O}^{k}\right)$ is shown. 

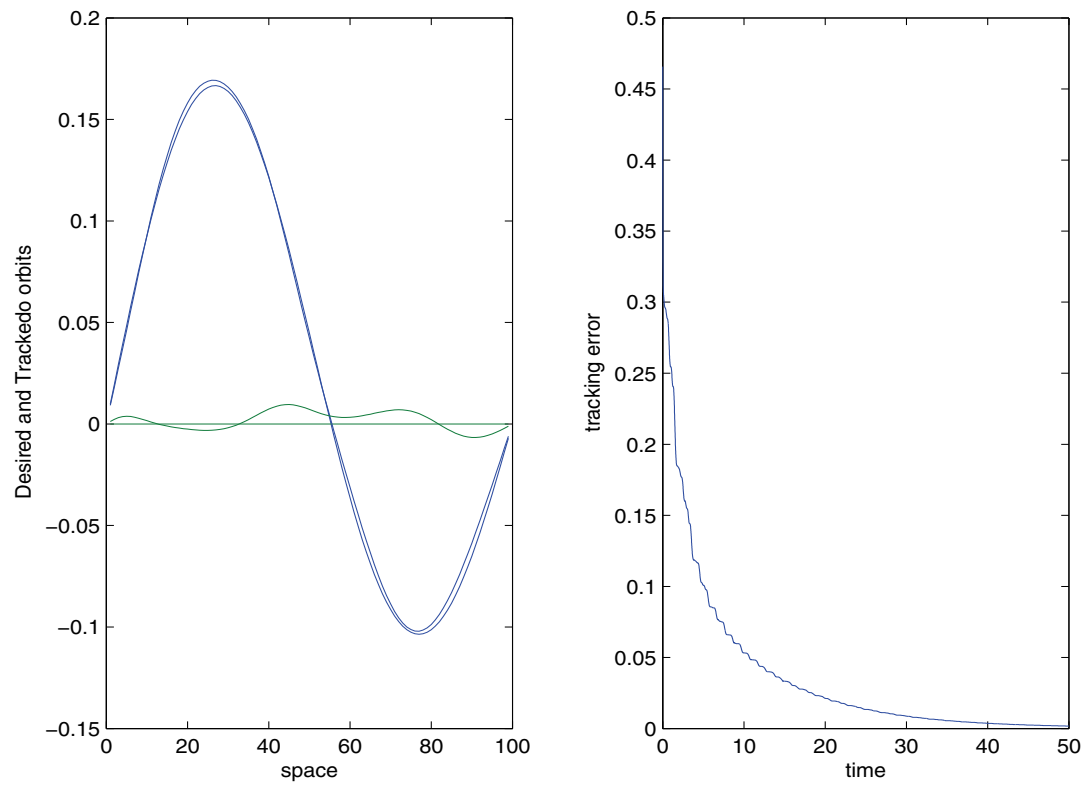

FIG. 1.

Acknowledgment. The authors express their appreciation to Prof. A. Borzi for discussions on various aspects of this paper.

\section{REFERENCES}

[A] C. Altafini, Feedback stabilization of isospectral control systems on complex flag manifolds: Application to quantum ensembles, IEEE Trans. Automat. Control, 52 (2007), pp. 2019-2028.

[C] A. Z. CAPRI, Nonrelativistic Quantum Mechanics, 3rd ed., World Scientific, Singapore, 2002.

[B] V. BARBU, Nonlinear Semigroups and Differential Equations in Banach Spaces, Noordhoff International Publishing, Leyden, 1976.

[BMS] J. M. Ball, J. E. Marsden, and M. Slemrod, Controllability for distributed bilinear systems, SIAM J. Control Optim., 20 (1982), pp. 575-597.

[BCMR] K. Beauchard, J. M. Coron, M. Mirrahimi, and P. Rouchon, Implicit Lyapunov control of finite dimensional Schrödinger equations, Systems Control Lett., 56 (2007), pp. 388395.

[CGRR] Y. Chen, P.Gross, V. Ramakrishna, and H. Rabitz, Competitive tracking of molecular objectives by quantum mechanics, J. Chem. Phys., 102 (1995), pp. 8001-8010.

[FK] R. A. Freeman and P. V. Kokotovic, Inverse optimality in robust stabilization, SIAM J. Control Optim., 34 (1996), pp. 1365-1391.

[G] S. T. GLAD, Robustness of nonlinear state feedback: A survey, Automatica, 23 (1987), pp. 425-435.

[I] A. E. Ingham, Some trigonometrical inequalities with applications to the theory of series, Math. Z., 41 (1936), pp. 367-379.

[IK] K. Ito AND F. Kappel, Evolution Equations and Approximations, World Scientific, River Edge, NJ, 2002.

[IK1] K. Iто AND K. Kunisch, Optimal bilinear control of an abstract Schrödinger equation, SIAM J. Control Optim., 46 (2007), pp. 274-287.

[M] M. Mirrahimi, Lyapunov control of a particle in a finite quantum potential well, in Proceedings of the 45th IEEE Decision and Control Conference, San Diego, CA, 2006, pp. 3962-3967.

[MRT] M. Mirrahimi, P. Rouchon, AND G. Turinici, Lyapunov control of bilinear Schrödinger

Copyright (c) by SIAM. Unauthorized reproduction of this article is prohibited. 
equations, Automatica J. IFAC, 41 (2005), pp. 1987-1994.

[P] A. PAZY, Semigroups of Linear Operators and Applications to Partial Differential Equations, Springer-Verlag, Berlin, 1983.

[S] M. SugawARA, General formulation of locally designed coherent control theory for quantum system, J. Chem. Phys., 118 (2003), pp. 6784-6800.

[T] R. Temam, Infinite-Dimensional Dynamical Systems in Mechanics and Physics, Appl. Math. Sci. 68, Springer-Verlag, Heidelberg, 1988.

[Yo] K. YosidA, Functional Analysis, Springer-Verlag, Berlin, 1980.

[Y] R. M. Young, An Introduction to Nonharmonic Fourier Series, Academic Press, New York, 1980.

[ZSR] W. ZhU, M. Smit, AND H. RABItz, Managing singular behavior in the tracking control of quantum dynamical systems, J. Chem. Phys., 110 (1999), pp. 1905-1915.

Copyright (c) by SIAM. Unauthorized reproduction of this article is prohibited. 